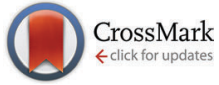

Cite this: Phys. Chem. Chem. Phys., 2015, 17, 4771

\section{Correction: Theoretical study and design of multifunctional phosphorescent platinum(II) complexes containing triarylboron moieties for efficient OLED emitters}

\author{
Yong Wu, Guo-Gang Shan, Hai-Bin Li, Shui-Xing Wu, Xin-Yao Ren, Yun Geng* and \\ Zhong-Min Su*
}

DOI: $10.1039 / c 4 c p 90185 a$

Correction for 'Theoretical study and design of multifunctional phosphorescent platinum(॥) complexes containing triarylboron moieties for efficient OLED emitters' by Yong Wu et al., Phys. Chem. Chem. Phys., 2015, DOI: 10.1039/c4cp04919e.

www.rsc.org/pccp

The authors regret that some values of the transition dipole moments, $\mu\left(\mathrm{S}_{n}\right)$, in Table 6 of the article are incorrect. The revised version of Table 6 is as shown below. The corrections of $\mu\left(\mathrm{S}_{n}\right)$ have no effect on the other calculations, discussions and conclusions.

Table 6 Transition dipole moments $\mu\left(\mathrm{S}_{n}\right)$ [Debye] for $\mathrm{S}_{0}-\mathrm{S}_{n}$ transitions, singlet-triplet splitting energies $\Delta E\left(\mathrm{~S}_{n}-\mathrm{T}_{1}\right)$ [eV] and the SOC matrix elements $\left\langle\mathrm{T}_{1}\left|\mathrm{H}_{\mathrm{sOC}}\right| \mathrm{S}_{n}\right\rangle\left[\mathrm{cm}^{-1}\right]$ of 1-6 at their respective $\mathrm{T}_{1}$ optimized geometries obtained from SOC-TD-B3LYP + COSMO calculations in $\mathrm{CH}_{2} \mathrm{Cl}_{2}$ solution

\begin{tabular}{|c|c|c|c|c|c|c|c|c|c|}
\hline $\mathrm{S}_{n}$ & \multicolumn{3}{|l|}{1} & \multicolumn{3}{|l|}{$\underline{2}$} & \multicolumn{3}{|l|}{3} \\
\hline $\mathrm{S}_{1}$ & 3.58 & 0.423 & 36.6 & 6.68 & 0.419 & 2.28 & 6.03 & 0.383 & 17.6 \\
\hline $\mathrm{S}_{3}$ & 1.32 & 0.919 & 145 & 1.83 & 1.275 & 86.5 & 2.09 & 0.995 & 127 \\
\hline $\mathrm{S}_{4}$ & 1.00 & 1.041 & 215 & 0.53 & 1.289 & 76.2 & 1.23 & 1.051 & 24.0 \\
\hline $\mathrm{S}_{5}$ & 0.66 & 1.070 & 739 & 0.78 & 1.402 & 76.4 & 1.04 & 1.194 & 373 \\
\hline & 4 & & & 5 & & & 6 & & \\
\hline $\mathrm{S}_{n}$ & $\mu\left(\mathrm{S}_{n}\right)$ & $\Delta E\left(\mathrm{~S}_{n}-\mathrm{T}_{1}\right)$ & $\left\langle\mathrm{T}_{1}\left|H_{\mathrm{SOC}}\right| \mathrm{S}_{n}\right\rangle$ & $\mu\left(\mathrm{S}_{n}\right)$ & $\Delta E\left(\mathrm{~S}_{n}-\mathrm{T}_{1}\right)$ & $\left\langle\mathrm{T}_{1}\left|H_{\mathrm{SOC}}\right| \mathrm{S}_{n}\right\rangle$ & $\mu\left(\mathrm{S}_{n}\right)$ & $\Delta E\left(\mathrm{~S}_{n}-\mathrm{T}_{1}\right)$ & $\left\langle\mathrm{T}_{1}\left|H_{\mathrm{SOC}}\right| \mathrm{S}_{n}\right\rangle$ \\
\hline $\mathrm{S}_{1}$ & 2.87 & 0.539 & 37.3 & 3.64 & 0.457 & 28.4 & 2.01 & 0.348 & 143 \\
\hline $\mathrm{S}_{2}$ & 4.76 & 0.742 & 33.4 & 1.67 & 0.690 & 297 & 3.04 & 0.519 & 273 \\
\hline $\mathrm{S}_{3}$ & 0.19 & 0.843 & 45.0 & 1.68 & 0.897 & 82.8 & 0.67 & 0.832 & 952 \\
\hline $\mathrm{S}_{4}$ & 3.15 & 1.051 & 25.3 & 1.73 & 1.008 & 97.9 & 0.59 & 0.972 & 168 \\
\hline
\end{tabular}

The Royal Society of Chemistry apologises for these errors and any consequent inconvenience to authors and readers. 